\title{
State of Water in Perfluorosulfonic Ionomer (Nafion) Proton Exchange Membranes
}

\author{
Z. Lu $^{\text {a,c, },}$, G. Polizos b,c ${ }^{\text {, E. Manias }}{ }^{\text {b,c,* }}$, and D. D. Macdonald ${ }^{\text {a,c,* }}$ \\ ${ }^{\text {a }}$ Center for Electrochemical Science \& Technology, \\ b Polymer Nanostructures Lab - Center for the Study of Polymeric Systems, \\ ${ }^{\mathrm{c}}$ Department of Materials Science \& Engineering, \\ Pennsylvania State University, University Park, Pennsylvania 16802, USA.
}

This manuscript reports on the direct identification of four states of water in hydrated Nafion ionomer polymers, including a quantitative measurement of their dynamics, as previously published in the Journal of the Electrochemical Society, 155, B163-B171 (2008). Here, we provide further details on the dynamics of these water states and we underline still-open scientific questions, towards stimulating further fundamental research in the field.

\section{Introduction}

Extensive investigations on perfluorinated ionomers (1-4) has been motivated from their increasing application in fuel cell devices. In particular, significant research effort has been focused on hydrated forms of acid-treated Nafion, a perfluorosulfonated ionomer used both as a separator and an electrolyte in proton exchange membrane (PEM) fuel cells. Since water facilitates the motion of protons through the polymer matrix (5-7), the water content and the water dynamics determine PEM properties such as proton conductivity, methanol and oxygen permeability, and electro-osmotic drag. Thus, it becomes imperative to understand the water nature, its location, and associated dynamics in PEM polymers, which motivated our prior work on water dynamics in Nafion and Flemion $(8,9)$.

Nafion, in its hydrated form, has phase separated morphology with nanometer sized hydrophilic and hydrophobic (ion rich and fluorocarbon) domains (10), facilitating a variety of geometric and physical ( $c f$. water/polymer interactions) environments where water exists $(8,9)$. Different water states have been proposed to exist in Nafion membranes -for a detailed review see for example Paddison et al. $(11,12)$ or Lu et al. $(8,9)$ and references therein- corresponding to different environments, and properly applied dielectric spectroscopic methods can directly map out the dynamics in at least four states of water $(8,9)$.

Here, we report a followup on a systematic dielectric relaxation spectroscopy study of hydrated Nafion 117 (8), aiming to further elucidate the dynamics and the nature of water in these membranes and put forward open questions that still remain unanswered.

\section{States of Water in Nafion}

The nature of water in the acid form of Nafion 117 (E.I. DuPont de Nemours, equivalent weight $E W=1100 \mathrm{~g} /$ equiv) was quantified at several hydration levels by dielectric relax-

\footnotetext{
${ }^{1}$ Present Address: Rochester Institute of Technology, Rochester, New York 14623, USA.

*Corresponding authors: EM via manias@psu.edu or DDM viaddm2@psu.edu
} 
ation spectroscopy (8). For that work, two independent experimental setups were used to collect complex dielectric permittivity spectra: broadband dielectric relaxation spectroscopy (BDRS) at low frequencies $\left[0.01 \mathrm{~Hz}-10 \mathrm{MHz}\right.$, at -80 to $\left.25^{\circ} \mathrm{C}\right]$ and transmission microwave DRS at higher frequencies [0.40-26 GHz, at 25 to $\left.45^{\circ} \mathrm{C}\right]$.

Two aspects for that research proved crucial:

i. Careful equilibration of the water content before and during the experiments (8); and

ii. Operation of the microwave DRS in the transmission mode $(8,9)$, rather than in the reflection mode.

Under these conditions, one can directly observe the states of water in PEM polymers, manifested through recording water populations with distinctly resolved dynamical behaviors, as well as how these dynamics change with temperature and hydration level:

The fastest process observed was identified (8) as the cooperative picosecond relaxation of free bulk-like water, whereas the slowest process (microsecond relaxation times) corresponds to water molecules strongly bound to the charged sulfonic groups (hydration shell of the sulfonates). A third type of water was also observed (8), also characterized by picosecond relaxation times, close to and about three times slower than those of bulk water, which was attributed to loosely bound water (8).

The dynamics of bulk-like water (free, isotropic) and of the loosely-bound water are in the picosecond region, which can be observed by microwave DRS, and are shown in Fig. 1 for Nafion at three hydration levels $\left(\lambda=3,6\right.$ and 9 , at $\left.25{ }^{\circ} \mathrm{C}\right)$ together with spectra from pure water. The much slower dynamics of the strongly bound water can be measured at the microsecond region via broadband DRS, and are shown in Fig. $2(\lambda=1$ and $\lambda=6)$.

Clearly, at the outset and without any data analysis necessary, there are at least three
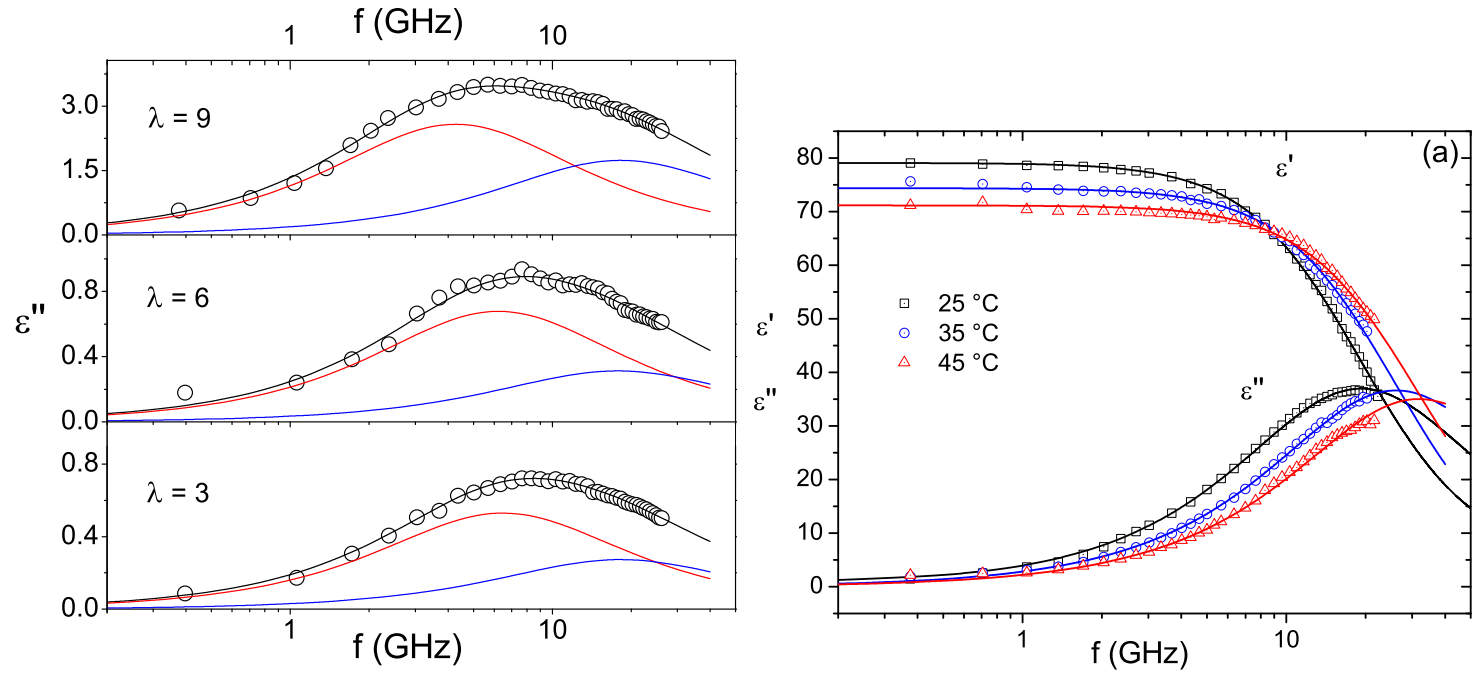

Figure 1: [left] Dielectric modes of water in hydrated Nafion $117\left(\lambda=3,6\right.$ and 9 , at $\left.25^{\circ} \mathrm{C}\right)$. The loss $\varepsilon^{\prime \prime}(f)$ relaxation distributions and their corresponding best fits, by two Debye processes, are shown. The faster processes (high frequencies, blue line) corresponds to bulk-like water in Nafion, and the slower process to loosely bound water (lower frequencies, red line). [right] Pure liquid water spectra under the same microwave dielectric relaxation transmission mode, the solid lines are again Debye relaxation fits; the real part of the permittivity $(\varepsilon(f))$ levels off at its bulk value (cf. water dielectric constant) below $\sim 2 G H z$ denoting that there are no appreciable losses with slower (sub-GHz) dynamics. Reproduced after permission from J. Electrochem. Soc., 155, B163 (2008), (c) 2008, The Electrochemical Society. 

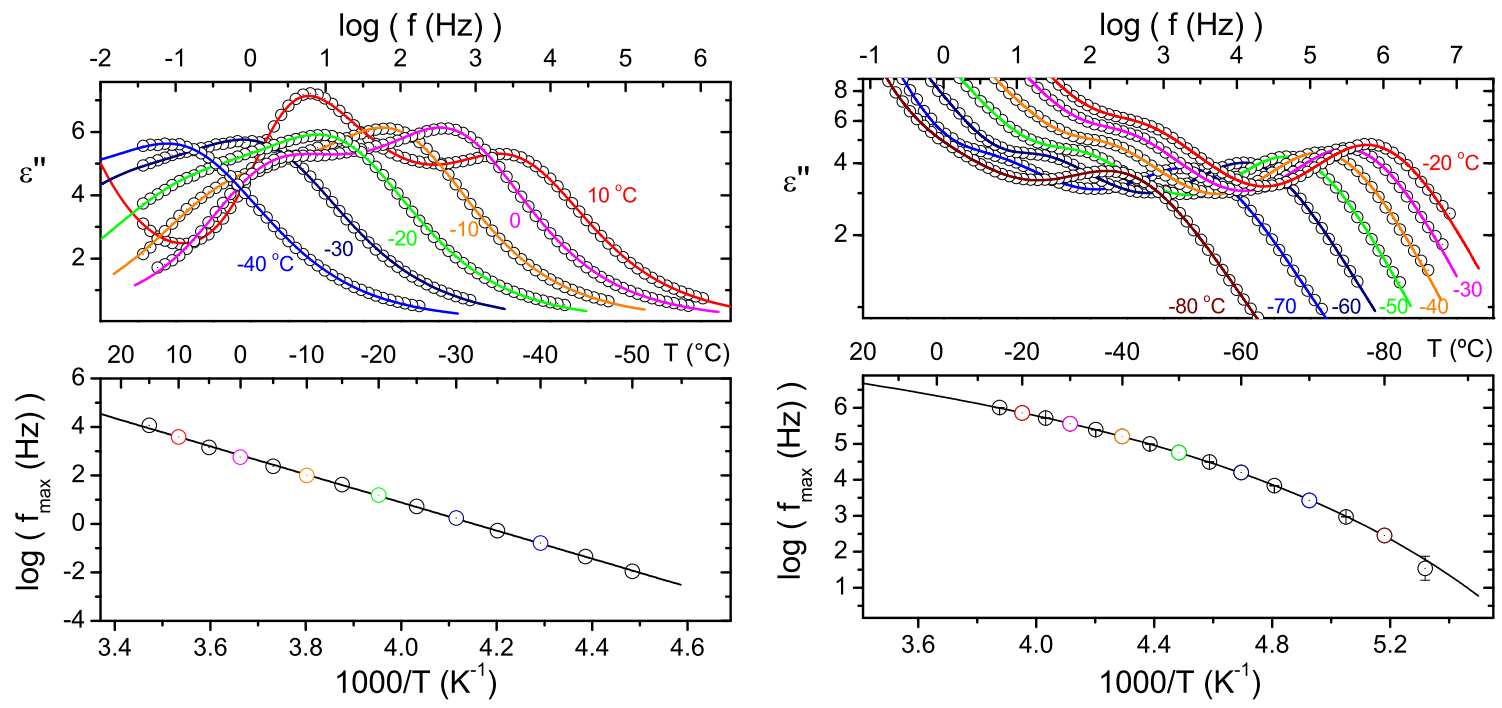

Figure 2: [left] Dielectric loss signals for Nafion 117 with $\lambda=1$ at low temperatures, the lines are Havriliak-Negami fits (eq. 1). The slower of the two modes is due to interfacial polarization effects (Maxwell-Wagner) and the faster mode is attributed to the rotation of water/sulfonate groups (first hydration shell). [bottom] Arrhenius plot of the peak frequency $\left(f_{\max }\right.$, relaxation rate) for the $\lambda=1$ bound water over all the temperatures studied, this relaxation clearly indicates a water that does not freeze. [right] Dielectric loss signal for Nafion with $\lambda=6$ at low temperatures, the lines are HaviliakNegami fits (eq. 1). The slower of the two modes is due to interfacial polarization effects (MaxwellWagner) and the faster mode is attributed to the rotation of water/sulfonate structures (first and second hydration shells, $\mathrm{SO}_{3}^{-}$and $\mathrm{H}_{3}^{+} \mathrm{O}$ complexes). (bottom) Arrhenius plot of the peak frequency $\left(f_{\max }\right)$ for the bound water relaxation over all the temperatures studied, this relaxation corresponds to water structures that undergo some kind of transition (for example, this data can be described rather well by a VTFH function, indicating an apparent glass transition at $168 \mathrm{~K}$ ). Reproduced after permission from J. Electrochem. Soc., 155, B163 (2008), (c) 2008, The Electrochemical Society.

populations of water in Nafion with distinctly different dynamics: two relaxations in the $p s$ range (Fig. 1) and a third relaxation in the $\mu s$ range (Fig. 2). However, identifying the nature of these water relaxations necessitates careful data analysis, e.g. see (8). Comparison with bulk liquid water spectra (13) clearly identifies the fastest of the three water populations as bulk-like water. Additionally, after closer inspection of the other data, the slower of the other two water states can also be definitively (8) identified as the water strongly-bound to the sulfonic acid groups (Fig. 2), via H-bonding and ion-dipole/dipole-dipole interactions; here, however, it is not clear what is the exact physical state of this water, since its dynamics are consistent both with amorphous supercooled water, as well as consistent with water that can freeze at a depressed temperature (vide infra).

Along the same lines, the slower of the two ps dynamics processes clearly corresponds to water molecules that are neither coordinated to sulfonic groups, nor do they exhibit unconstrained, bulk-like, liquid water dynamics. This type of water can be envisioned in various environments within the Nafion membrane, but for the hydration levels considered in these experiments it can be argued that it would consist mostly of "loosely bound" water (8), i.e., water at the polymer interfaces, or a second hydration shell surrounding the water coordinated to the sulfonates, or, most probably (8, see footnote $d)$, waters that reside in the hydrophilic paths connecting between the hydrated ionic clusters or at the interfaces 
Table I: . Parameters from fitting of the BDRS data of Fig. 2 ( $\lambda=1$, 'dry' Nafion 117) with equation 1 , there were no conductivity contributions in the raw data. The first peak is due to interfacial polarization effects (charge build up), and the second peak $\left(f_{\circ, 2}, \Delta \varepsilon_{2}\right)$ corresponds to the relaxation of the $\mathrm{SO}_{3}^{-}$/water structures. $\chi^{2}$ is the standard least square fit criterion (with $\chi^{2}=0$ indicating a perfect fit to the data) and it relates to the more usual fitting correlation coefficient $R$, which is 0.999 or better for all fits shown here.

\begin{tabular}{|c|c|c|c|c|c|c|c|c|c|}
\hline $\mathrm{T}(\mathrm{K})$ & $\alpha_{1}$ & $\beta_{1}$ & $f_{\circ, 1}(\mathrm{~Hz})$ & $\Delta \varepsilon_{1}$ & $\alpha_{2}$ & $\beta_{2}$ & $f_{\circ, 2}(\mathrm{~Hz})$ & $\Delta \varepsilon_{2}$ & $\chi^{2}$ \\
\hline 288 & $0.10 \pm 0.01$ & $0.43 \pm 0.01$ & $3.89 \pm 0.06$ & $32.23 \pm 0.19$ & $0.44 \pm 0.01$ & 1.0 & $11754 \pm 243$ & $14.38 \pm 0.22$ & 0.00141 \\
\hline 283 & $0.13 \pm 0.01$ & $0.32 \pm 0.01$ & $1.93 \pm 0.05$ & $29.00 \pm 0.44$ & $0.39 \pm 0.01$ & 1.0 & $3632 \pm 207$ & $14.50 \pm 0.45$ & 0.00132 \\
\hline 278 & $0.26 \pm 0.01$ & $0.36 \pm 0.02$ & $1.22 \pm 0.04$ & $26.93 \pm 0.59$ & $0.39 \pm 0.01$ & 1.0 & $1430 \pm 18$ & $15.90 \pm 0.50$ & 0.00101 \\
\hline 268 & $0.39 \pm 0.01$ & $0.47 \pm 0.04$ & $0.46 \pm 0.04$ & $22.28 \pm 1.23$ & $0.41 \pm 0.01$ & 1.0 & $238 \pm 5.7$ & $18.19 \pm 1.06$ & 0.00215 \\
\hline 263 & $0.44 \pm 0.01$ & $0.50 \pm 0.03$ & $0.26 \pm 0.02$ & $22.31 \pm 0.97$ & $0.41 \pm 0.01$ & 1.0 & $101 \pm 2.8$ & $18.29 \pm 0.82$ & 0.00216 \\
\hline 248 & - & - & - & - & $0.44 \pm 0.01$ & 1.0 & $5.34 \pm 0.05$ & 20.00 fixed & 0.00147 \\
\hline 243 & - & - & - & - & $0.45 \pm 0.01$ & 1.0 & $1.73 \pm 0.01$ & 21.00 fixed & 0.00155 \\
\hline 238 & - & - & - & - & $0.45 \pm 0.01$ & 1.0 & $0.53 \pm 0.01$ & 22.00 fixed & 0.00144 \\
\hline 233 & - & - & - & - & $0.45 \pm 0.01$ & 1.0 & $0.16 \pm 0.01$ & 22.80 fixed & 0.00047 \\
\hline 228 & - & - & - & - & 0.45 fixed & 1.0 & $0.04 \pm 0.0003$ & 23.50 fixed & 0.00028 \\
\hline
\end{tabular}

Table II: Parameters from fitting of the BDRS data of Fig. 2 ( $\lambda=6$, 'hydrated' Nafion 117) with equation 1 after substraction of the conductivity contributions [not shown here]. The two peak attributions and the parameter definitions are as before (Table I).

\begin{tabular}{|c|c|c|c|c|c|c|c|c|c|}
\hline $\mathrm{T}(\mathrm{K})$ & $\alpha_{1}$ & $\beta_{1}$ & $f_{\circ, 1}(\mathrm{~Hz})$ & $\Delta \varepsilon_{1}$ & $\alpha_{2}$ & $\beta_{2}$ & $f_{\circ, 2}(\mathrm{~Hz})$ & $\Delta \varepsilon_{2}$ & $\chi^{2}$ \\
\hline 258 & $0.10 \pm 0.05$ & $0.34 \pm 0.05$ & $392.03 \pm 9.59$ & $8.92 \pm 0.40$ & $0.33 \pm 0.01$ & 1.0 & $1014590 \pm 10756$ & $14.31 \pm 0.20$ & 0.00152 \\
\hline 253 & $0.17 \pm 0.04$ & $0.42 \pm 0.04$ & $320.14 \pm 19.61$ & $12.06 \pm 0.76$ & $0.35 \pm 0.01$ & 1.0 & $721924 \pm 9085$ & $14.76 \pm 0.29$ & 0.00109 \\
\hline 248 & $0.15 \pm 0.05$ & $0.41 \pm 0.05$ & $255.85 \pm 17.84$ & $11.30 \pm 0.79$ & $0.34 \pm 0.01$ & 1.0 & $519718 \pm 7531$ & $14.28 \pm 0.33$ & 0.00144 \\
\hline 243 & $0.15 \pm 0.04$ & $0.41 \pm 0.04$ & $202.73 \pm 13.32$ & $10.25 \pm 0.56$ & $0.33 \pm 0.01$ & 1.0 & $362437 \pm 4452$ & $13.79 \pm 0.27$ & 0.00102 \\
\hline 238 & $0.10 \pm 0.03$ & $0.37 \pm 0.04$ & $151.67 \pm 9.14$ & $8.83 \pm 0.41$ & $0.32 \pm 0.01$ & 1.0 & $247243 \pm 2742$ & $13.22 \pm 0.26$ & 0.00088 \\
\hline 233 & $0.10 \pm 0.04$ & $0.37 \pm 0.05$ & $114.15 \pm 8.70$ & $8.26 \pm 0.41$ & $0.33 \pm 0.01$ & 1.0 & $160237 \pm 2175$ & $13.00 \pm 0.30$ & 0.00117 \\
\hline 228 & $0.10 \pm 0.04$ & $0.33 \pm 0.04$ & $65.70 \pm 4.63$ & $9.10 \pm 0.35$ & $0.32 \pm 0.01$ & 1.0 & $99211 \pm 1110$ & $12.35 \pm 0.32$ & 0.00089 \\
\hline 223 & $0.10 \pm 0.04$ & $0.33 \pm 0.04$ & $44.34 \pm 3.44$ & $9.02 \pm 0.37$ & $0.33 \pm 0.01$ & 1.0 & $57449 \pm 741$ & $12.20 \pm 0.38$ & 0.00102 \\
\hline 218 & $0.17 \pm 0.03$ & $0.36 \pm 0.04$ & $23.80 \pm 1.97$ & $10.97 \pm 0.36$ & $0.33 \pm 0.01$ & 1.0 & $31285 \pm 393$ & $11.91 \pm 0.40$ & 0.00079 \\
\hline 213 & $0.15 \pm 0.04$ & $0.22 \pm 0.02$ & $13.44 \pm 0.72$ & $10.75 \pm 0.70$ & $0.32 \pm 0.01$ & 1.0 & $16046 \pm 205$ & $10.46 \pm 0.45$ & 0.00153 \\
\hline 208 & $0.15 \pm 0.04$ & $0.33 \pm 0.06$ & $6.70 \pm 0.64$ & $11.70 \pm 0.65$ & $0.35 \pm 0.01$ & 1.0 & $6813 \pm 110$ & $11.03 \pm 0.67$ & 0.00101 \\
\hline 203 & $0.15 \pm 0.02$ & $0.27 \pm 0.03$ & $2.35 \pm 0.18$ & $14.00 \pm 0.81$ & $0.34 \pm 0.02$ & 1.0 & $2679 \pm 268$ & $9.77 \pm 0.61$ & 0.00145 \\
\hline 198 & $0.17 \pm 0.02$ & $0.29 \pm 0.02$ & $1.15 \pm 0.06$ & $13.50 \pm 0.51$ & $0.36 \pm 0.01$ & 1.0 & $931 \pm 10$ & $9.88 \pm 0.31$ & 0.00030 \\
\hline 193 & $0.24 \pm 0.03$ & $0.34 \pm 0.02$ & $0.44 \pm 0.08$ & $13.92 \pm 0.81$ & $0.37 \pm 0.01$ & 1.0 & $282 \pm 5$ & $9.63 \pm 0.35$ & 0.00027 \\
\hline
\end{tabular}

between the polymer and the bulk-like liquid water ( $c f$. hydrophobic hydration).

Thus, based on the dynamical data at hand, the exact nature of either of these two water populations remain as open questions. Before we venture into addressing these questions, we will briefly mention the fitting method and provide the fitting results for the DRS data. We used the superposition of two Havriliak-Negami expressions (14-16) to fit the data, with:

$$
\varepsilon^{*}(\omega)=\varepsilon_{\infty}+\frac{\Delta \varepsilon_{1}}{\left[1+\left(i \omega / 2 \pi f_{\circ, 1}\right)^{1-\alpha_{1}}\right]^{\beta_{1}}}+\frac{\Delta \varepsilon_{2}}{\left[1+\left(i \omega / 2 \pi f_{\circ, 2}\right)^{1-\alpha_{2}}\right]^{\beta_{2}}}-i \frac{\sigma_{\circ}}{\varepsilon_{\circ}} \omega^{-s}
$$

$\varepsilon_{\infty}=3.03 \pm 0.01$ for $\lambda=1$ and $\varepsilon_{\infty}=3.06 \pm 0.01$ for $\lambda=6$; the conductivity exponent $s$ is less than 1, i.e., it is not a genuine dc conductivity, therefore, the conductivity prefactor $\sigma_{\circ}$ is not reported below. This expression (eq. 1) naturally fits the two peak response usually observed in these systems, but can also be used with single peak data where it degenerates to a single peak [depending on the initial conditions: either by converging to zero value for 
one of the two dielectric strengths $(\Delta \varepsilon)$, or by converging to a single relaxation rate for the two peaks $\left.\left(f_{\circ, 1}=f_{\circ, 2}\right)\right]$.

\section{Open Questions on the Water States in Nafion}

$\underline{\text { Strongly bound water: Supercooled amorphous, or water with depressed freezing point? }}$

The strongly bound water behavior in hydrated Nafion (e.g. $\lambda=6$ ) at low temperatures $\left(-10\right.$ to $\left.-90{ }^{\circ} \mathrm{C}\right)$ posses a very interesting question on the specific nature of this water state. Specifically, the measured response of this water state could correspond to either a supercooled (amorphous) water that exhibits a glass transition (17-19), or to a water that exhibits a dramatically reduced freezing temperature (e.g. due to H-bonding, dipole-dipole interactions, and nanoscale confinement).

For the 'dry' Nafion (Table I), the loss peak maximum frequency peak at $f_{\max }=f_{\circ}$ is a symmetric peak ( $\beta=1$, Cole-Cole distribution) and, thus, $f_{\max }(T)$ was fitted by an Arrhenius equation:

$$
f_{\text {max }}=f_{\infty} \exp \left(-\Delta E_{A} / k_{B} T\right)
$$

This fit provides an activation energy of $\Delta E_{A}=106 \pm 1 \mathrm{~kJ} / \mathrm{mol}$ for $\lambda=1$ (Table I, peak 2 ); where, $f_{\infty}$ is the relaxation rate in the high temperature limit and $k_{B}$ the Boltzmann constant. The Arrhenius plot of the $\varepsilon^{\prime \prime}$ peak frequency $\left(f_{\max }\right.$, shown in Fig. 2$)$ reveals no change in the activation energy, denoting that the water associated with this process does not undergo a phase change, i.e., it does not form a crystalline structure, and it clearly corresponds to the non freezable water in Nafion; i.e., its dynamics are in excellent agreement with the strongly bound first hydration shell of the $\mathrm{SO}_{3}$ groups in Nafion (20-23).

The temperature dependence of the loss peak frequency maximum $f_{\max }$ for $\lambda=6 \mathrm{can}$ be fitted rather well (Fig. 3) either with two Arrhenius responses or with a single VTFH response (see details below). Although mathematically either of the two fits is equally acceptable (and very close to the experimental data points), the physics ( $c f$. nature of this water) are completely different is the two case:

1. The data can be fit with two Arrhenius equations (eq. 2), yielding activation energies of $\Delta E_{A}=39 \pm 1.0 \mathrm{~kJ} / \mathrm{mol}$ for the high temperatures and $\Delta E_{A}=69 \pm 1.5 \mathrm{~kJ} / \mathrm{mol}$ for the low temperatures, with a transition at about $217 \mathrm{~K}\left(-56{ }^{\circ} \mathrm{C}\right)$, which would denote a crystallization transition for the sulfonate/water clusters, i.e., a depressed freezing temperature for water due to H-bonding and confinement; or

2. Alternatively, and mathematically equally well, the same data can be fit with a single VTFH equation throughout the complete temperature range:

$$
f_{\text {max }}=A \exp \left(-\frac{B}{T-T_{\circ}}\right)
$$

where $A, B$ and $T_{\circ}$ are temperature independent empirical constants; the Vogel temperature $T_{\circ}$ is the ideal glass transition temperature, which has been found in many glass-forming polymers to be about 40-50 K lower than the $T_{g}$. The fit for peak (2) of for $\lambda=6$ gives $\log A=9.03 \pm 0.06$, $(A$ in $H z), B=840.67 \pm 22.74 \mathrm{~K}$, and $T_{\circ}=137.66 \pm 1.03 \mathrm{~K}$, with excellent fit quality $\left(\chi^{2}=0.00014\right.$, viz. $\left.R^{2}=0.99991\right)$; the $T_{g}$ that corresponds to this fit (by extrapolation to $f_{\max }=0.0016 \mathrm{~Hz}$ or $\tau=100 \mathrm{~s}$ ) is $168 \mathrm{~K}$ $\left(-105^{\circ} \mathrm{C}\right)$, in excellent agreement with the $T_{g}$ of supercooled water $(17,18)$. 


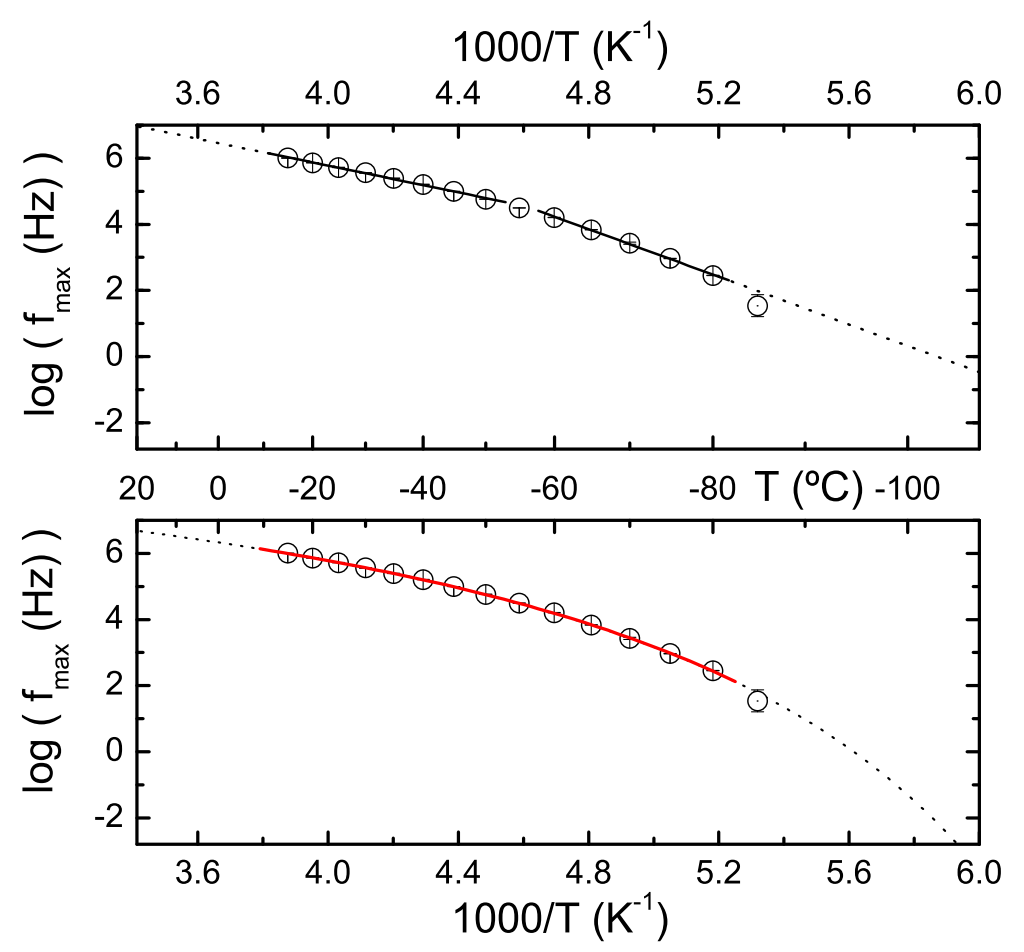

Figure 3: Comparative fits of the temperature dependence of the dielectric loss peak frequency maximum $f_{\max }$ for the Broadband DRS of the $\lambda=6$ Nafion membranes [data shown in Table s.II, peak (2)]: (top) two Arrhenius lines, (bottom) a single VTFH curve. The datum at $-85^{\circ} \mathrm{C}$ was not used for any of the fits, the solid denote the range of values where the fit was done, whereas the dotted lines are extrapolations of the fits. For the VTFH fit, the $T_{g}$ corresponds to the extrapolated temperature at $f_{\max }=0.0016 \mathrm{~Hz}\left(\log f_{\max }=-2.79818\right.$ or $\tau=100 \mathrm{~s})$ and is $168 \mathrm{~K}\left(-105^{\circ} \mathrm{C}\right)$.

The comparison of these two fits is shown in Figure 3. Despite the excellent agreement of the extrapolated $T_{g}$ from our DRS data to the typical $T_{g}$ of supercooled water (as well as the other VTFH parameters all being reasonably close to those of supercooled water), it is impossible to dismiss the possibility of this water being a confinement-affected crystallizable structure with a depressed freezing temperature. In addition, further experiments carried out in our lab towards further identifying this water were inconclusive: For example, DSC did not measure an endothermic response upon cooling (would have been proof of a depressed water crystallization) and FTIR did not identify any peaks that would point to any type of ice; however, considering the resolution of both these techniques and the expected population of the corresponding water, these results may simply be due to insufficient experimental sensitivity. The best, most promising, way to answer this question is probably via atomistic molecular simulations, e.g. through analysis of Molecular Dynamics trajectories $(24,25)$.

Loosely bound water: Interfacial water, or hydrophobic hydration?

The loosely bound water relaxation time ranges between 20 and $40 \mathrm{ps}$, depending on the Nafion's water content and temperature, in excellent quantitative agreement with wa- 
ter loosely bound to aliphatic alcohols and carboxylic acids (26) and other hydrophobic molecules (27-29). Comparable relaxation times for water, e.g. $\tau_{\max }=16-30 \mathrm{ps}$, have also been reported for aqueous solutions of micelles (30) and proteins (31), and were attributed to water molecules surrounding the micellar or protein surface and interacting weakly with them, $c f$. loosely bound water. However, for Nafion this type of water would reside in many different local environments that are inherently heterogeneous in their dynamics; thus, at first glance, it is striking that excellent fits of this loosely bound water dynamics can be obtained by a single Debye peak, denoting a well-defined single relaxation time dynamical mode. In our previous work (8), we showed that this single relaxation time (monoexponential) mode observed for loosely bound water was a result of our sample preparation procedure, which allowed for the development of well equilibrated morphologies within Nafion (8). Nevertheless, the origins of a single Debye relaxation time mechanism in water observed via DRS are still not clear, not even in the simpler case of bulk/liquid water (32); for example, it has been argued that there are at least two molecular relaxations contributing to such behavior: a single-molecule rotation mechanism and a "tetrahedral displacement" -including both an antisymmetric stretching and a torsion mode- that can together account for the single water relaxation time (32).

One way to make this dynamic heterogeneity manifest in DRS spectra was shown in

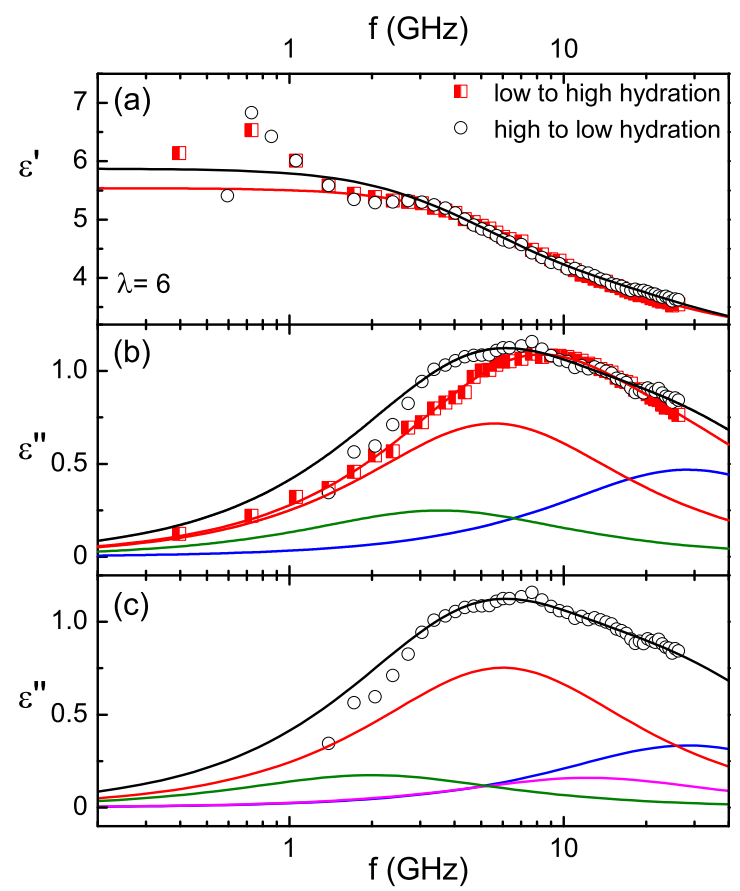

Figure 4: Permittivity spectra at $\lambda=6$ and $45^{\circ} \mathrm{C}$ for two different hydration approaches: Squares correspond to well-equilibrated morphologies formed by slow water sorption (low-to-high $\lambda$ change); whereas, circles correspond to less-well-equilibrated morphologies obtained by drying (high-to-low $\lambda$-change, water desorption). Although the membrane's water content was set, in both cases, to $\lambda=6$, there exist clear differences in the dielectric response, especially in the shape of the dielectric losses, $\varepsilon^{\prime \prime}$, which clearly shows more-than-two Debye processes (circles, desorption), whereas a single Debye peak is seen for the equilibrated morphologies (squares, sorption). Reproduced with permission from J. Electrochem.Soc.,155, B163 (2008), (c) The Electrochemical Society. 
(8); specifically, by altering the sample preparation approach, we reached less-equilibrated Nafion/water morphologies, characterized by the same hydration levels $(\lambda=6)$. This was done by starting with highly hydrated membranes $(\lambda>12)$ and drying them, by heating and/or under vacuum, removing water until the desired $\lambda$ was reached. For these systems, the loosely bound Nafion water showed definitively multiple distinct dynamical modes (Fig. 4).

As mentioned above, loosely bound water can exist, by definition, in many different local environments that are inherently heterogeneous in their dynamics. For example, the measured dynamics are consistent with several different polymer environments:

1. one water environment with a distribution of sizes, e.g. water in hydrophilic channels of varied diameters and lengths; or

2. a water environment with a gradual or continuous distribution of dynamics, e.g. extended hydrophilic domains wherein the water exhibits dynamics depended on its location, accelerating from slow dynamics near the polymer/water interface to bulklike water dynamics when well inside the free water domain; or, most probably:

3. a variety of different environments that are characterized by similar/overlapping dynamics, e.g. hydrophilic channels, water/polymer interfaces of extended hydrophilic domains, second/third hydration shell(s) around the sulfonate/water cluster, water confined in the hydrophobic domains of the membrane, etc.

Dielectric spectroscopy methods alone do not have enough fidelity to distinguish between all the above options. This would necessitate a membrane hydration protocol that would reproducibly result in the same metastable/transient water environment every time, and high fidelity spectra to yield unambiguous fits, and multiple well-separated temperatures for the microwave DRS (our instrument can only cover a limited range of $25-50{ }^{\circ} \mathrm{C}$ ) to identify Arrhenius activation energies for each of the Debye peaks. Again, atomistic Molecular Dynamics simulations $(11,12,24,25,33,34)$ seem to be the best choice to further probe this line of research.

\section{Conclusions}

When proper (transmission mode) microwave dielectric spectroscopy is applied to hydrated Nafion and complemented by lower frequency (broadband) dielectric techniques, then the dynamics of the various water states can be quantitatively mapped-out. However, there remain open questions on the exact nature of these water states, which cannot be addressed experimentally by DRS. Here, we show that Nafion's strongly-bound water is consistent with both crystallizable confined water (with a depressed freezing point), as well as with amorphous supercooled water (with the usual $T_{g}$ for amorphous water). Also, the nature of the loosely-bound water remains uncertain and could be attributed to one or more of several possible water environments that develop inside a polymer ionomer PEM. 


\section{Acknowledgements}

The authors gratefully acknowledge support from International Fuel Cells Inc. (subcontract no. 3540OB), US Department of Energy (contract no. DE-FC04-02AL67608),

the National Science Foundation (grant no. DMR-0602877), and the Office of Naval Research (grant no. 00014-05-1-0614).

\section{References}

[1] M. A. F. Robertson and H. L. Yeager, Structure and properties of perfluorinated ionomers (Chapman \& Hall, 1997), chap. 7, Ionomers, synthesis, structure, properties and applications.

[2] A. D. I. Eisenberg and J. S. Kim, Introduction to ionomers (John Wiley \& Sons, Inc, 1998).

[3] M. Rikukawa and K. Sanui, Prog. Polym. Sci. 25, 1463 (2000).

[4] K. A. Mauritz and R. B. Moore, Chem. Rev. 104, 4535 (2004).

[5] M. A. Hickner and B. S. Pivovar, Fuel Cells 5, 213 (2005).

[6] M. A. Hickner, N. P. Siegel, K. S. Chen, et al. J. Electrochem. Soc. 153, A902 (2006).

[7] M. A. Hickner, C. H. Fujimoto, and C. J. Cornelius, Polymer 47, 4238 (2006).

[8] Z. Lu, G. Polizos, D. D. Macdonald, and E. Manias, J. Electrochem. Soc. 155, B163 (2008).

[9] Z. Lu, M. Lanagan, E. Manias, and D. D. Macdonald, J. Phys. Chem. B 113, 13551 (2009).

[10] R. B. Moore and C. R. Martin, Macromolecules 22, 3594 (1989).

[11] S. J. Paddison, Annu. Rev. Mater. Res. 33, 289 (2003).

[12] K. D. Kreuer, S. J. Paddison, E. Spohr, and M. Schuster, Chem. Rev. 104, 4637 (2004).

[13] W. J. Ellison, J. Phys. Chem. Ref. Data 36, 1 (2007).

[14] S. Havriliak and S. Negami, Polymer 8, 161 (1967).

[15] N. G. McCrum, B. E. Read, and G. Williams, Anelastic and Dielectric Effects in Polymeric Solids (Dover Publications Inc., NY, 1967).

[16] F. Kremer and A. Schönhals, eds., Broadband Dielectric Spectroscopy (Springer, 2002).

[17] V. Velikov, S. Borick, and C. A. Angell, Science 294, 2335 (2001).

[18] P. G. Debenedetti and F. H. Stillinger, Nature 410, 259 (2001).

[19] P. G. Debenedetti and H. E. Stanley, Physics Today 56, 40 (2003).

[20] M. Cappadonia, J. W. Erning, and U. Stimming, J. Electroanalytical Chem. 376, 189 (1994).

[21] S. A. Perusich, P. Avakian, and M. Y. Keating, Macromolecules 26, 4756 (1993).

[22] C. Tsonos, L. Apekis, and P. Pissis, J. Mater. Sci. 35, 5957 (2000).

[23] C. Tsonos, L. Apekis, and P. Pissis, J. Mater. Sci. 33, 2221 (1998).

[24] V. Kuppa, S. Menakanit, R. Krishnamoorti, et al. J. Polym. Sci. Polym. Phys. 41, 3285 (2003).

[25] E. Manias and V. Kuppa, Europ. Phys. J. E 8, 193 (2002).

[26] K. Hellenga, J. R. Grlgera, and H. J. C. Berendsen, J. Phys. Chem. 84, 2381 (1980).

[27] U. Kaatze and A. Rupprecht, J. Chem. Phys. 117, 4936 (2002).

[28] Y. E. Ryabov, Y. Feldman, N. Shinyashiki, and S. Yagihara, J. Chem. Phys. 116, 8610 (2002).

[29] A. Lileeva, D. Loginova, A. Lyashchenko, et al. J. Molec. Liq. 131-132, 101 (2007).

[30] C. Baar, R. Buchner, and W. Kunz, J. Phys. Chem. B 105, 2906 (2001).

[31] N. Nandi and B. Bagchi, J. Phys. Chem. B 101, 10954 (1997).

[32] N. Agmon, J. Phys. Chem. 100, 1072 (1996).

[33] J. A. Elliott and S. J. Paddison, Phys. Chem. Chem. Phys. 9, 2602 (2007).

[34] E. Allahyarov, P. L. Taylor, H. Löwen, Phys. Rev. E 80,061802 (2009), ibid, 81,031805 (2010). 